\title{
Erratum: TET1 is a tumor suppressor of hematopoietic malignancy
}

Luisa Cimmino, Meelad M Dawlaty, Delphine Ndiaye-Lobry, Yoon Sing Yap, Sofia Bakogianni, Yiting Yu, Sanchari Bhattacharyya, Rita Shaknovich, Huimin Geng, Camille Lobry, Jasper Mullenders, Bryan King, Thomas Trimarchi, Beatriz Aranda-Orgilles, Cynthia Liu, Steven Shen, Amit K Verma, Rudolf Jaenisch \& Iannis Aifantis

Nat. Immunol. 16, 653-662 (2015); published online 13 April 2015; corrected after print 17 June 2015

In the version of this article initially published, labels reading " $5 \mathrm{hmC}$ gain" were incorrectly included below the plots in Figure $6 \mathrm{e}$, and the plot at right was mislabeled above (as "loss"). The plot at left should have a single label above reading "5hmC loss" and the plot at right should have a single label above reading " $5 \mathrm{hmC}$ gain." The error has been corrected in the HTML and PDF versions of the article.

\section{Erratum: The diverse role of RIP kinases in necroptosis and inflammation}

John Silke, James A Rickard \& Motti Gerlic

Nat. Immunol. 16, 930-936 (2016); published online 18 June 2015; corrected after print 26 June 2015

In the version of this article initially published, the following were incorrect and should be corrected as stated here. In Fig 1 , ref. 84 should be ref. 82 , and "pers. comm." should be cited one line below (aligned with $M l \mathrm{kl}^{-{ }^{-}}$). The middle of the third sentence of the Fig. 2 legend should include not a period but a comma, to read "phosphorylation of RIPK3, which leads to" and the end of fifth sentence should not include "RIPK" but should read "which leads to RIPK1-independent necroptosis...." The fifth sentence of final paragraph of the subsection "RIPK1-, RIPK3- and MLKL-induced necroptosis" should not include "cIAP" but should begin "First, mice deficient in both cIAP1 and cIAP2...." The middle of the sixth sentence of first paragraph in the subsection "Evidence for necroptotic DAMPS" should not state this as "concentration" but should read "however, HMGB1 levels are not higher" and final sentence should include citation of ref. 49 to read "...do not ${ }^{49}$ ". In Fig. 4, the arrow from pro-IL- $1 \beta$ should be a solid line, not a dashed line. The third sentence of the first paragraph of the subsection "RIP kinases and MLKL as therapeutic targets" should cite ref. 74 instead of ref. 76 (to read "mutation ${ }^{74}$."), and the final sentence of that subsection should be deleted. Fig. 5 should include the following revisions: in the top right section (Brain), the text should not include a parenthetical element but read "brain injury ${ }^{117,118}$, controlled cortical impact trauma ${ }^{119}$ and"; in the upper left section (Skin), the first sentence should not end with "RIPK" but should end "RIPK1 (refs. 48,49,57)"; in the middle left section (Liver), "no effect on" should be orange in both places (not only the first); and in the bottom section (Systemic), the second line should read "TNFand Z-VAD-induced hyper-acute shock" (not "TNF- or Z-VAD-"). The errors have been corrected in the HTML and PDF versions of the article. 